\title{
The Construction Method of the QRS Loop in Myocardial Infarction
}

\author{
Seiichi Toyama, M.D. and Keiko Suzukr, M.D.
}

\section{SUMMARY}

In order to investigate the relation between the infarcted area in myocardial infarction and the change of the QRS loop, the following method was tried.

The normal QRS loops in 3 planes were constructed with the digital computer. Then the vector of an optional segment corresponding to infarction was diminished and the QRS loops were constructed. The comparison of the QRS loops in clinical cases and in the construction method was made and the vectorcardiographic findings to estimate the infarcted area were discussed.

\section{Additional Indexing Words:}

Myocardial infarction High posterior infarction
Construction method of the QRS loop Anterior infarction with LAH

$\mathbf{I}^{\mathrm{N}}$

$\mathrm{N}$ cases with myocardial infarction, it is important to determine the area of the infarcted myocardium with the electrocardiogram or the vectorcardiogram. The abnormal $Q$ wave or the deformity of the QRS loop contributes to diagnose the location of myocardial infarction.

However, there have been few reports of a detailed comparison of vectorcardiographic findings and autopsy, and in order to determine the vectorcardiographic criteria of myocardial infarction, the electrocardiographic study has been widely used. Therefore, the diagnostic reliability of the vectorcardiogram could not be superior to that of the electrocardiogram.

In another method, the model of the QRS loop was constructed and a comparison of the QRS loop between clinical cases and that model was made by some investigators. Toyoshima et al $^{1)}$ described the QRS loop in their re-construction method, and Sevlester ${ }^{21,3)}$ also reported the model of the QRS loop with an analog and a digital computer. Recently, Okajima ${ }^{4}$ has made an elaborated model with a digital computer.

The authors have tried to construct the QRS loop with a digital computer. In this method, the vector of an optional segment was diminished due to myocardial infarction and the QRS loops were drawn on the $\mathrm{X}-\mathrm{Y}$ plotter

From the Department of Cardiology, Center for Adult Diseases, Osaka, 3-3 Nakamichi 1-chome, Higashinari-ku, Osaka 537, Japan.

Received for publication August 8, 1975. 
following calculation with a digital computer. Then, the authors have compared the QRS loop in clinical cases and that in the construction method, and discussed the vectorcardiographic findings to determine the infarcted area with the QRS loop.

\section{METHOD}

In the construction method, the left and right ventricles including the ventricular septum were divided into 14 segements; the segments of the left and right sides in the ventricular septum (LS and RS), and the inferior and superior segments of the anterior, lateral, and posterior walls in both ventricles (Lai, Las, Lmi, Lms, Lpi, Lps, Rai, Ras, Rmi, Rms, Rpi, and Rps) as shown in Fig. 1.

The location of the ventricular septum was at 60 degrees in the horizontal plane. The ventricular depolarization time was $80 \mathrm{msec}$. Gauss' curve was used for the contribution of the depolarization process in each segment of the ventricles. The direction of the vector in each segment was assumed as shown with direction cosines in Table I. The maximal magnitude of each segment in the left ventricle was 3 times larger than that in the right ventricle. Those values were in-put into the computer of NEAC 2200-150 and after calculation, the QRS loops in the horizontal, left sigittal and frontal planes were drawn with the X-Y plotter. Thereafter, the vector of an optional segment which corresponded to the infarcted area was diminished to zero, and the QRS loops in 3 planes were drawn.

In clinical cases with myocardial infarction whose vectorcardiograms were recorded, typical cases were selected and compared to the QRS loop of the construction method.



Horizontal plane



Sagittal plane

Fig. 1. Segments of the ventricular septum and left and right ventricles. (Lai = the inferior segment of the anterior wall in the left ventricle ...). 
Table I. Maximal Amplitude and Direction Cosine of the Vector in Each Segment

\begin{tabular}{l|l|l|r|r|r}
\hline Segment & A & B & L & M & \multicolumn{1}{|c}{ N } \\
\hline LS & 3.5 & 10 & -0.866 & 0.000 & 0.500 \\
RS & 2.5 & 10 & 0.837 & 0.259 & -0.483 \\
Lai & 3.0 & 30 & 0.866 & 0.000 & 0.500 \\
Las & 3.0 & 40 & 0.750 & 0.500 & 0.433 \\
Lmi & 3.0 & 40 & 0.750 & -0.500 & -0.433 \\
Lms & 3.0 & 50 & 0.837 & 0.259 & -0.483 \\
Lpi & 3.0 & 40 & 0.000 & -0.866 & -0.500 \\
Lps & 3.0 & 50 & 0.000 & -0.259 & -0.966 \\
Rai & 1.0 & 30 & 0.000 & 0.000 & 1.000 \\
Ras & 1.0 & 40 & 0.000 & 0.500 & 0.866 \\
Rmi & 1.0 & 40 & -0.750 & -0.500 & 0.433 \\
Rms & 1.0 & 50 & -0.837 & 0.259 & 0.483 \\
Rpi & 1.0 & 40 & -0.433 & -0.866 & -0.250 \\
Rps & 1.0 & 50 & -0.837 & -0.259 & -0.483
\end{tabular}

A : maximal amplitude of the segment,

B : the time (msec) of the amplitude,

$\mathrm{L}, \mathrm{M}$, and $\mathrm{N}$ : direction cosine.

Duration of each segment except LS and RS is $60 \mathrm{msec}$.

a)

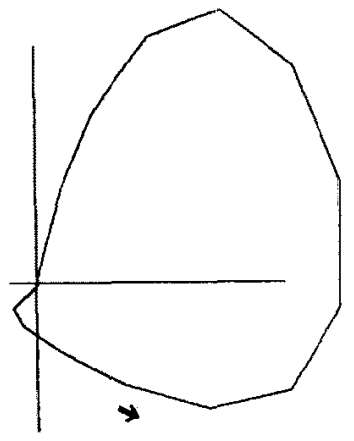

b)

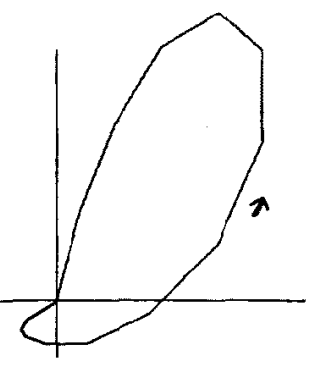

H.P.


L.S.P.

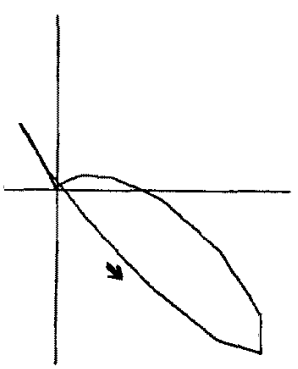

F.P.

Fig. 2. a) Normal QRS loops in 3 planes. (H.P. = horizontal plane, L.S.P. = left sagittal plane, F.P. $=$ frontal plane, and in this order in Figs. $2 \sim 8$ ).

b) QRS loops when the vector of Lai was diminished to zero. 


\section{REsults}

1) Normal QRS loop

The QRS loops in Fig. 2-a were normal configurations in the authors' construction method.

\section{2) Anterior infarction}

Assuming that the inferior segment of the anterior wall in the left ventricle was infarcted, the vector of Lai was diminished to zero. The horizontal QRS loop was initiated right-anteriorly, but the left anterior portion was dislocated more posteriorly than that of the normal QRS loop (Fig. 2-b).

When the vectors of Lai and Rai were zero due to myocardial infarction of the anterior wall in both ventricles, the initial QRS loop in the horizontal plane was directed right-anteriorly, but the efferent limb was dislocated leftposteriorly. When the vectors of Lai, Rai, LS, and RS were zero due to anteroseptal infarction, the whole QRS loop in the horizontal plane was dislocated left-posteriorly (Fig. 3-a) and similar to that in the clinical case of Fig. 3-b.

a)

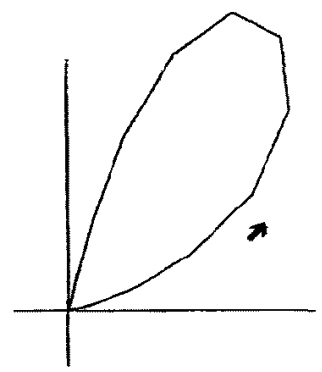



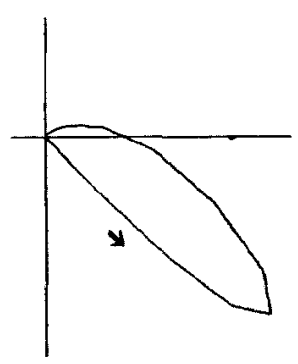

b)

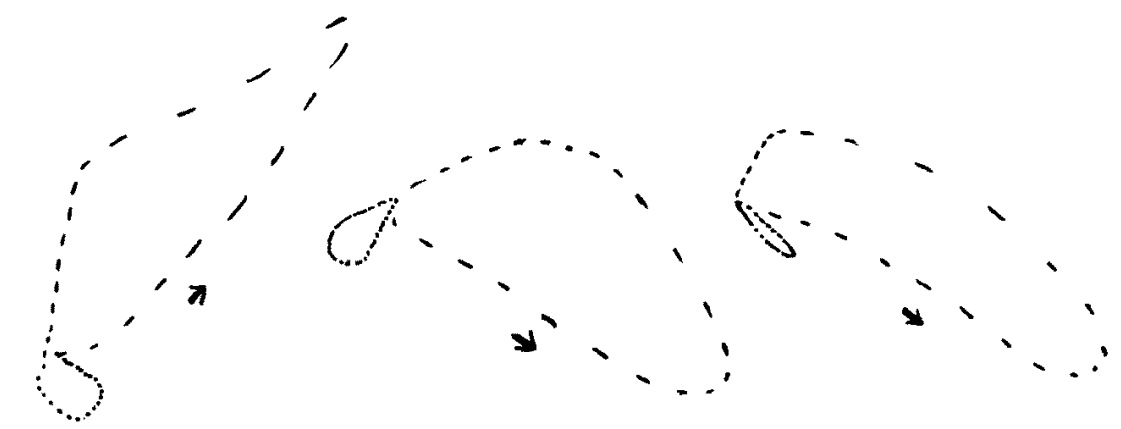

Fig. 3. a) QRS loops when the vectors of Lai, Rai, LS, and RS were zero.

b) A clinical case of anterior infarction. 
a)


b)



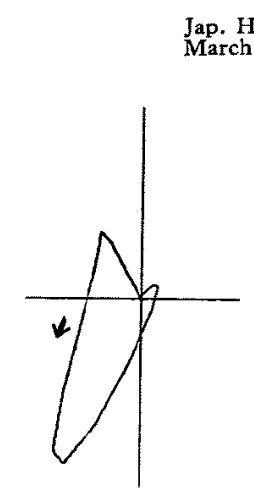

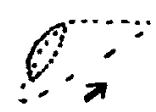



Fig. 4. a) QRS loops when the vectors of Lai, Las, and Lmi were zero.

b) A clinical case of anterolateral infarction.

a)



b)

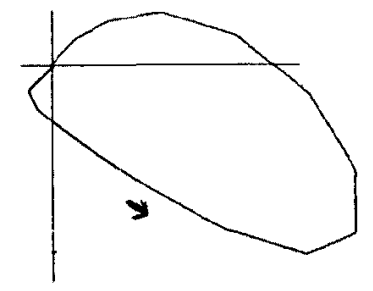

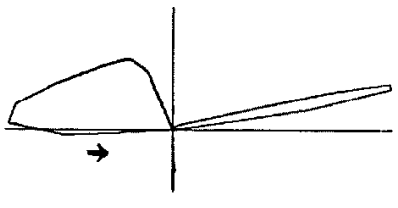
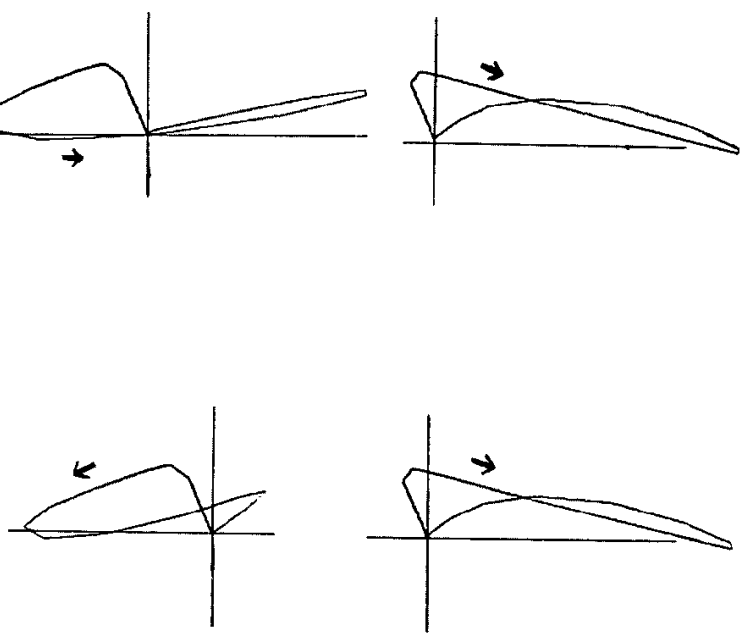

Fig. 5. a) QRS loops when the vector of Lpi was zero.

b) QRS loops when the vectors of $L$ pi and $L$ ps were zero. 


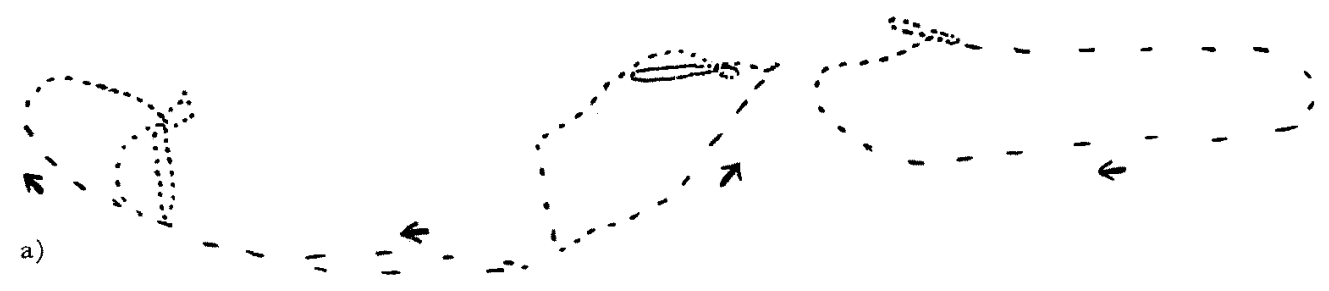

b)


Fig. 6. Clinical cases of high posterior infarction.

a) Type I, b) Type II.

a)



b)



c)

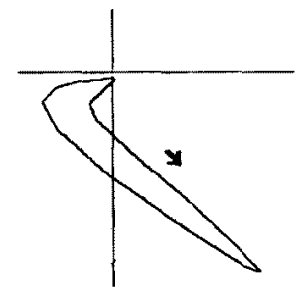

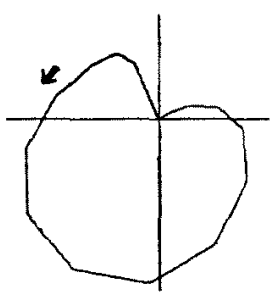
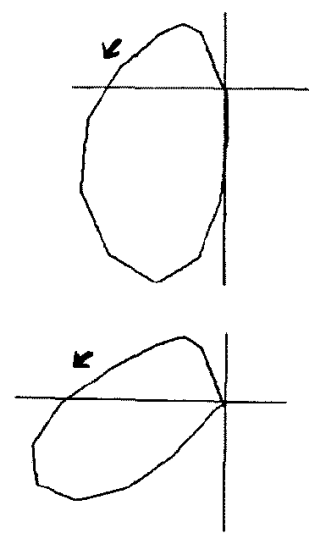


Fig. 7. a) QRS loops when the vector of Lps was zero.

b) QRS loops when the vectors of $L p s$ and Lms were zero.

c) QRS loops when the vectors of $\mathrm{Lps}$, $\mathrm{Lms}$, and $\mathrm{Lmi}$ were zero. 
3) Anterolateral infarction

When the vectors of Lai, Las, and Lmi were zero due to anterolateral infarction, the horizontal QRS loop was initiated right-anteriorly and the efferent limb was located right-posteriorly. That QRS loop was similar to that in the clinical case of Fig. 4-b.

4) Inferior infarction

In clinical cases with inferior infarction, there was no significant difference of the horizontal QRS loop between before and after infarction. In the construction method, when the vector of Lpi was zero due to inferior infarction, the efferent limb of the frontal QRS loop was dislocated left-superiorly, but the configuration of the horizontal QRS loop was as same as that of the normal QRS loop (Fig. 5-a). Moreover, when the vector of Lps was zero due to the extension of the infarction, the afferent limb of the horizontal QRS loop was dislocated anteriorly (Fig. 5-b).

5) High posterior infarction

In clinical cases, the authors divided the configurations of the horizontal

a)

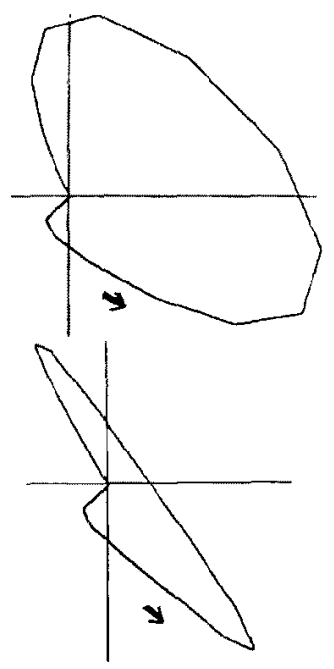

c)

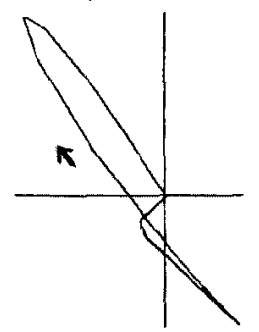

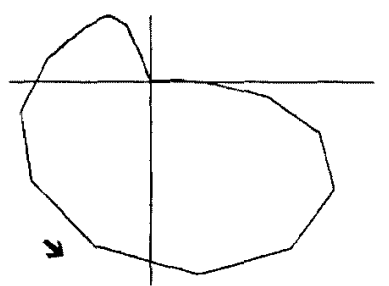
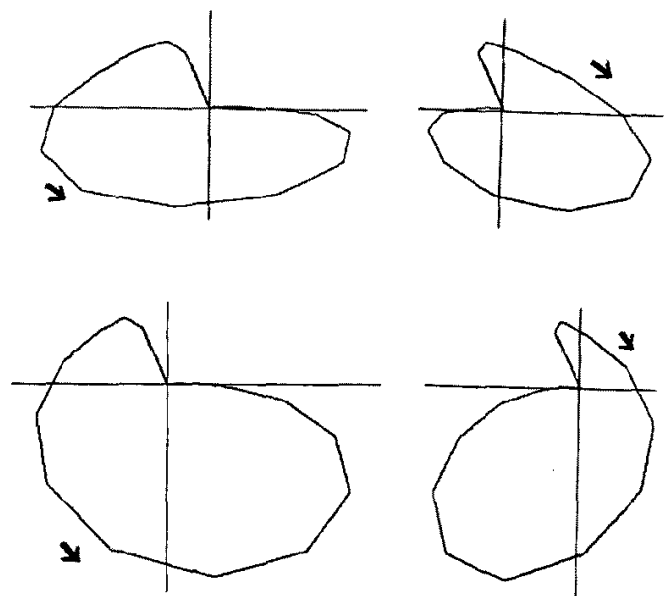
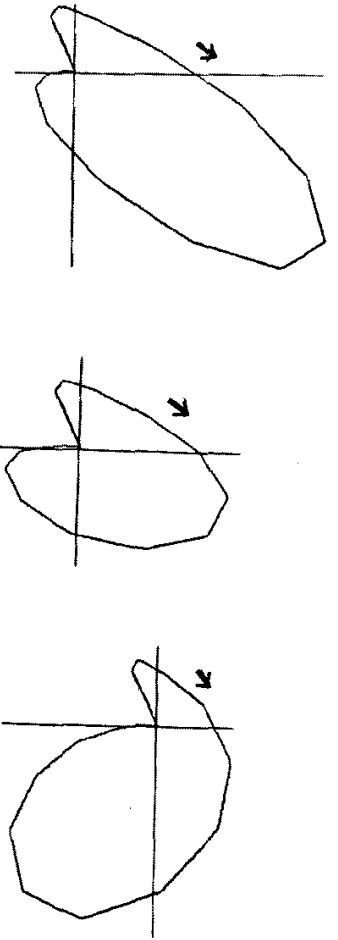

Fig. 8. a) QRS loops when the vector of Lms was zero.

b) QRS loops when the vectors of $L m s$ and $L m i$ were zero.

c) QRS loops when the vectors of $\mathrm{Lms}, \mathrm{Lmi}$, and Las were zero. 
QRS loop into 2 types. The horizontal QRS loop in Type I was pushed from the posterior side and dislocated anteriorly (Fig. 6-a). That in Type II was pushed from the left-posterior side and located from left-anteriorly to rightposteriorly (Fig. 6-b).

When the vector of Lps was zero due to localized infarction of the high posterior wall in the left ventricle, the posterior portion of the horizontal $Q R S$ loop was diminished (Fig. 7-a). When the vectors of Lps and Lms were zero due to high posterolateral infarction, the horizontal QRS loop was dislocated anteriorly and similar to that of Type I (Fig. 7-b). When the vectors of Lps, Lms and Lmi were zero, the horizontal QRS loop was dislocated more rightanteriorly with clockwise inscription and the initial QRS loop in the frontal plane was displaced superiorly (Fig. 7-c).

When the vector of Lms alone was zero, the left-posterior portion of the horizontal QRS loop was cut (Fig. 8-a), but such a configuration of the QRS loop was often observed in normal cases. When the vectors of Lms and Lmi were zero, the horizontal QRS loop was dislocated from left-anteriorly to right-posteriorly (Fig. 8-b). When the vectors of Lms, Lmi, and Las were zero, the horizontal QRS loop was dislocated more right-anteriorly with inscription of Fig. 8, and similar to that of Type II (Fig. 8-c).

\section{Discussion}

The abnormal $Q$ wave in the electrocardiogram is useful in determining the location of myocardial infarction. Recently, it has been said that a decreasing amplitude of the $\mathrm{R}$ wave is also helpful to diagnose infarction. In the vectorcardiogram, the deformity or the dislocation of the QRS loop is a diagnostic sign of infarction. But, the vectorcardiographic criteria for diagnosing infarction are usually based on the abnormal $Q$ wave in the electrocardiogram, because it is difficult to establish the criteria with a comparison between vectorcardiographic findings and autopsy. However, with this method, the vectorcardiogram cannot be more beneficial than the electrocardiogram.

In another method, a model of the QRS loop is constructed and compared to that in clinical cases. Toyoshima et al ${ }^{11}$ used their reconstruction method and discussed the reason of the dislocation of the QRS loop in myocardial infarction. Selvester ${ }^{2,3)}$ reported the method with a digital computer and described the deformity of the QRS loop in small and large infarctions. Okajima and his coworkers ${ }^{4}$ made the model of the ventricle composed of a cluster of $3 \mathrm{~mm}$ cubic blocks. In their study, a ventricular propagation process was simulated, and the QRS complex and vector loop were reconstructed 
with the digital computer HITAG 5020E. Kohno') analyzed the reason of the abnormal $Q$ wave in subendocardial infarction with that method.

The authors' construction method is similar to Selvester's method, but the ventricular septum and free walls are different in location. The time history and the direction of the vector in each segment are also determined from the authors' standpoint. Regarding this method, there are many kinds of assumption with many problems. However, such problems are common in the construction method of a model, and the QRS loop similar to that in a normal case can be obtained with this method. Moreover, as the QRS loops in right and left ventricular hypertrophy and left anterior hemiblock could be simulated, this construction method is used in the present study.

In this method, the vector of one segment is diminished to zero, when the corresponding segment is infarcted. In anterior infarction, when the vector of the inferior segment of the anterior wall in the ventricle (Lai) is zero, the efferent limb of the horizontal QRS loop is dislocated slightly posteriorly, but remains in the left anterior quadrant. However, when the vectors of Lai and Rai are zero, the horizontal QRS loop is initiated right-anteriorly, but the efferent limb is dislocated left-posteriorly. Besides, when the vectors of the ventricular septum (LS and RS) are zero, the whole $Q R S$ loop in the horizontal plane is displaced left-posteriorly and is similar to that in a clinical case with anteroseptal infarction. Namely, the constructed QRS loop with a digital computer is coincident with the QRS loop in clinical cases. In antero-lateral and inferior infarction, it is also shown that both QRS loops are very similar to those in clinical cases. In the infarction of the inferior segment of the posterior wall in the left ventricle (Lpi), the efferent and afferent limbs of the frontal QRS loop are displaced superiorly, but no significant change is found in the horizontal QRS loop like the finding in a clinical case. However, when the infarcted area extends to the superior segment of the posterior wall (Lps), the posterior portion of the horizontal QRS loop is dislocated anteriorly, and such QRS loop is often observed in inferior infarction combined with so-called high posterior infarction.

In so-called high posterior infarction, the authors have distinguished 2 types depending on the location of the horizontal QRS loop. The horizontal QRS loop of Type I is located anteriorly. In the construction method, the QRS loop similar to that of Type $I$ is obtained when the superior segments of the posterior and lateral walls in the left ventricle (Lps and Lma) are infarcted. On the other hand, the horizontal QRS loop of Type II is dislocated to left-anteriorly and right-posteriorly, and that QRS loop is obtained when the lateral wall in the left ventricle (Lms and Lmi) is mainly infarcted. When the superior segment of the anterior wall in the left ventricle (Las) is involved, 
the horizontal QRS loop is displaced more right anteriorly. Therefore, it can be said that the difference between Type I and Type II is depending on the infarcted area.

The authors have reported that there were 2 kinds of so-called periinfarction block. One was the left anterior or posterior hemiblock with myocardial infarction, the other a delayed excitation of the subepicardial side with subendocardial infarction. In anterior infarction, the afferent limb of the QRS loop in the former was dislocated left-anterosuperiorly and the direction of inscription of the horizontal QRS loop is a figure of eight or clockwise. The QRS loop in the former can be constructed with the authors' method. The afferent limb of the horizontal QRS loop is located left-anteriorly and that of the frontal QRS loop left-superiorly. Namely, that afferent limb in this method is similar to that in some clinical cases with anterior infarction. Therefore, it is supported that the left-anterosuperior dislocation of the afferent limb in cases with anterior infarction is caused by a delayed excitation of the upper area of infarction due to left anterior hemiblock.

Of course, it is necessary to compare the QRS loops in the construction method and in clinical cases with autopsy findings in a future study. However, as mentioned above, it is helpful in determining the area of myocardial infarction to analyze the deformity or the dislocation of the QRS loop with the authors' construction method.

\section{References}

1. Toyoshima $H$, Kato $H$, Ishibe $T$, Kutsuna $Y$, Nagaya $T$, Saruhashi $Y$ : Electrocardiogram and vectorcardiogram reconstruction and its application to clinical diagnosis of myocardial infarction. Am Heart J 56: 165, 1958

2. Selvester RH, Collier CR, Pearson RB: Analog computer model of the vectorcardiogram. Girculation 31: 45, 1965

3. Selvester RH, Kalaba R, Collier CR, Bellman R, Kagiwada $\mathbf{H}$ : A digital computer model of the vectorcardiogram with distance and boundary effects; Simulated myocardial infarction. Am Heart J 74: 792, 1967

4. Okajima M, Fujino T, Kobayashi T, Yamada K: Computer simulation of the propagation process in excitation of the ventricles. Circulat Res 23: 203, 1967

5. Kohno M: QRS patterns in anterior subendocardial infarction-By means of simulation of propagation process and computational QRS reconstruction. Jap Circulat J 34: 981, 1970 